\title{
Compact maximal hypersurfaces in globally hyperbolic spacetimes
}

\author{
Juan A. Aledo ${ }^{a}$, Rafael M. Rubio ${ }^{b}$ and Juan J. Salamanca ${ }^{c}$ \\ ${ }^{a}$ Departamento de Matemáticas, E.S.I. Informática, \\ Universidad de Castilla-La Mancha, 02071 Albacete, Spain, \\ E-mail: juanangel.aledo@uclm.es \\ ${ }^{b},{ }^{c}$ Departamento de Matemáticas, Campus de Rabanales, \\ Universidad de Córdoba, 14071 Córdoba, Spain, \\ E-mails: rmrubio@uco.es, jjsalamanca@uco.es
}

\begin{abstract}
Several uniqueness results on compact maximal hypersurfaces in a wide class of globally hyperbolic spacetimes are provided. They are obtained from the study of a distinguished function on the maximal hypersurface and under suitable natural first order conditions of the spacetime. As a consequence, several applications to Geometric Analysis are given.
\end{abstract}

Keywords: Compact spacelike hypersurface, maximal hypersurface, globally hyperbolic spacetime.

PACS 2010: 02.40.Ky, 04.20.Cv.

\section{Introduction}

On a spacetime, a continuos function which is strictly increasing on any future directed causal curve, is called a time function. In 1968, Hawking proved that any stably causal spacetime admits a time function [18]. Recall that a causal spacetime is said stably causal, if there is a fine $C^{0}$ neighborhood of the original metric of the spacetime such that any of its Lorentzian metrics is causal [7]. Conversely, Hawking also proved that a spacetime is stably causal if it admits a smooth function with an everywhere timelike gradient. More recently, Bernal and Sánchez [8] defined a temporal function as a smooth function on the spacetime with (past-directed) timelike gradient on all the spacetime, and proved that any spacetime which admits a time function also admits a temporal function (see also [29]). Thus, as the authors highlighted, this result combined with Hawking's ones, ensures, on one hand, that any stably causal spacetime admits a temporal function and, on the other hand, that any spacetime which admits a time function is stably causal.

There exist a serie of folk questions referred to differentiability of time functions and Cauchy hypersurfaces, and smooth orthogonal splitting of globally hyperbolic spacetimes. The main theorem in [8] assures that an $(n+1)$-dimensional globally hyperbolic spacetime 
$M$ is isometric to a smooth product manifold

$$
\mathbb{R} \times \mathcal{S}, \quad \bar{g}=-\beta d \mathcal{T}^{2}+\hat{g},
$$

where $\mathcal{S}$ is a smooth spacelike Cauchy hypersurface, $\mathcal{T}: \mathbb{R} \times \mathcal{S} \rightarrow \mathbb{R}$ the natural projection, $\beta: \mathbb{R} \times \mathcal{S} \rightarrow(0, \infty)$ a smooth function, and $\hat{g}$ a 2-covariant symmetric tensor field on $\mathbb{R} \times \mathcal{S}$, satisfying:

i) $\nabla \mathcal{T}$ is timelike and past-pointing on all $M$.

ii) Each hypersurface $\mathcal{S}_{\mathcal{T}}$ at constant $\mathcal{T}$ is a Cauchy hypersurface, and the restriction $\hat{g}_{\mathcal{T}}$ of $\hat{g}$ to $\mathcal{S}_{\mathcal{T}}$ is a Riemannian metric.

iii) The radical of $\hat{g}$ at each $w \in \mathbb{R} \times \mathcal{S}$ is $\operatorname{Span} \nabla \mathcal{T}$ at $w$.

If we denote by $\pi_{\mathcal{S}}$ the canonical projection on $\mathcal{S}$, which is a diffeomorphism restricted to each level hypersurface $\mathcal{S}_{\mathcal{T}}$, via $\pi_{\mathcal{S}}$ we can give an one-parameter family of Riemannian metrics $g_{\mathcal{T}}$ on the differentiable manifold $\mathcal{S}$.

Inspired in all these facts, we consider a natural family of globally hyperbolic spacetimes which provides a kind setting for studying maximal hypersurfaces.

Consider a differentiable manifold $F$, an open interval $I \subset \mathbb{R}$ and an one-parametric family $\left\{g_{t}\right\}_{t \in I}$ of Riemannian metrics on $F$, i.e., a smooth map $\Lambda: I \times F \rightarrow T_{0,2}(F)$, where $T_{0,2}(F)$ denotes the fiber bundle of 2-covariant tensors on $F$, such that $\Lambda_{t}: F \rightarrow T_{0,2}(F)$ is a positive definite metric tensor for all $t \in I$. The product manifold $M=I \times F$ can be endowed with the Lorentzian metric given at each point $(t, p) \in I \times F$ by

$$
\bar{g}=\beta \pi_{I}^{*}\left(-d t^{2}\right)+\pi_{F}^{*}\left(g_{t}\right) \quad\left(\bar{g}=-\beta d t^{2}+g_{t} \text { in short }\right),
$$

where $\pi_{I}$ and $\pi_{F}$ denote the canonical projections onto $I$ and $F$, respectively, and $\beta \in$ $C^{\infty}(I \times F)$ is a positive function.

We will say that a spacetime is orthogonal-splitted, if it is isometric to a spacetime $(M, \bar{g}=$ $\left.-\beta d t^{2}+g_{t}\right)$. Observe that every orthogonal-splitted spacetime is stably causal.

Maximal hypersurfaces play a relevant role in General Relativity and Lorentzian Geometry by several reasons. Among them, it should be emphasized that this class of hypersurfaces are important tools in the analysis of the Cauchy problem with the purpose of dealing with simpler constraint equations or solve them (see [13], [14] and [15, Chap. VI]). Also, in the proof of positivity of gravitational mass, maximal hypersurfaces appear as useful geometric objects (see [31]). Furthermore, they describe the transition between expanding and contracting phases of the universe, in some relevant cases [9]. A complete summary of reasons justifying the importance of maximal (and constant mean curvature) hypersurfaces in General Relativity can be found in [21].

From a mathematical point of view, the maximal hypersurfaces arise naturally as critical points of the $n$-dimensional area functional for compactly supported normal variations. A global and motivating result concerning maximal hypersurfaces is the Calabi-Bernstein theorem. This theorem asserts that the only complete maximal hypersurfaces in the LorentzMinkowski spacetime, $\mathbb{L}^{n}$, are the spacelike hyperplanes. This fact was proved by Calabi [11] for $n \leq 4$, and extended to arbitrary dimension by Cheng and Yau [12]. In another spacetimes, the problem of characterizing maximal hypersurfaces has become a research topic of a great interest. For instance, in [10] Brill and Flaherty replaced the Minkowski spacetime with a spatial closed universe, and proved uniqueness at large by assuming $\operatorname{Ric}(z, z)>0$ for all 
timelike vectors $z$ (ubiquitous energy condition). In [21], this energy condition was relaxed by Marsden and Tipler to include, for instance, non-flat vacuum spacetimes. On the other hand, Bartnik [6] proved very general existence theorems and, consequently, he claimed that it would be useful to find new satisfactory uniqueness results. More recently, in [5] Alías, Romero and Sánchez proved new uniqueness results in the class of spatially closed generalized Robertson-Walker spacetimes (which includes the spatially closed Robertson-Walker spacetimes), under the Temporal Convergence Condition. Moreover, in [4] Alías and Montiel improved some of these results and, employing a generalized maximum principle due to Omori [22] and Yau [33], gave a general uniqueness result for the case of complete constant mean curvature spacelike hypersurfaces under certain boundedness of the Ricci curvature. Finally, in [26] Romero, Rubio and Salamanca obtained uniqueness results in the maximal case for spatially parabolic generalized Robertson-Walker spacetimes, which are open models whose fiber have a parabolic universal Riemannian covering (see [26] and [27]). Recall that a generalized Robertson-Walker (GRW) spacetimes, introduced in [5], is given by the product manifold of an interval of the real line, $I$, and a Riemannian manifold $\left(F, g_{F}\right)$ furnished with the Lorentzian metric $\bar{g}=-d t^{2}+f^{2} g_{F}$, where $f: I \longrightarrow(0, \infty)$ is a smooth function. Another rencent results about uniqueness of constant mean curvature spacelike complete (maximal and of constant mean curvature) hypersurfaces in GRW spacetimes can be found in [1], [2], [24] and [28].

The main aim of this work is to study uniqueness of compact maximal hypersurfaces in orthogonal-splitted spacetimes which satisfy certain natural first order conditions, in particular avoiding curvature assumptions. Thus, in Proposition 1 a characterization of orthogonalsplitted spacetimes which admit a compact spacelike hypersurface (i.e., the spacetime is spatially closed) is provided. We also prove that, under certain natural hypotheses, the compact maximal hypersurfaces must be level hypersurfaces of the time function.

In Section 3, we assume certain homogeneity in the expansive or contractive behavior of the orthogonal-splitted spacetime. More specifically, for each $v \in T_{q} F, q \in F$, denote by $\tilde{v}$ the lift of $v$ on the integral curve $\alpha_{q}(s)=(s, q) \in I \times F$. We say that the spacetime $(M, \bar{g})$ is non-contracting in all directions if $\partial_{t} \beta \leq 0$ and $\partial_{t} \bar{g}(\tilde{v}, \tilde{v}) \geq 0$, for all $q \in F$ and $v \in T_{q} F$. Analogously, we say that the spacetime is non-expanding in all directions if the previous inequalities are reversed. Observe that the spacetime is non-contracting (resp. nonexpanding) if and only if $\mathcal{L}_{\partial_{t}} \bar{g}$ is semi-definite positive (resp. semi-definite negative), where $\mathcal{L}$ denotes the Lie derivative.

If we consider the observer field $U=\frac{1}{\sqrt{\beta}} \partial_{t}$, the proper time $\tau$ of the observers in $U$ is given by $d \tau=\sqrt{\beta} d t$. As a consequence, the assumption $\partial_{t} \beta \leq 0$ (resp. $\partial_{t} \beta \geq 0$ ) assures that the rate of change (acceleration) of the proper time with respect to the time function $t$ is non-increasing (resp. non-decreasing). Observe also that the Lorentzian length $\left|\partial_{t}\right|=-\sqrt{\beta}$ is non-decreasing (resp. non-increasing) along the integral curves of $\partial_{t}$. All these physical interpretations can be considered locally.

On the other hand, the spacelike assumption $\partial_{t} \bar{g}(\tilde{v}, \tilde{v}) \geq 0\left(\right.$ resp. $\left.\partial_{t} \bar{g}(\tilde{v}, \tilde{v}) \leq 0\right)$, guarantees that an observer in $U$ measures non-contraction (resp. non-expansion) in all directions of its physical space.

There are many important examples of orthogonal-splitted spacetimes satisfying these conditions. For instance, any standard static spacetime $M=I \times F, \bar{g}=-\beta d t^{2}+g$, being $\beta: F \longrightarrow(0, \infty)$ a smooth function, clearly obeys our assumptions. Also a GRW spacetime satisfies the imposed conditions whenever the derivative $f^{\prime}$ of the warping function is signed. 
As a last example, let us consider the differentiable manifold $M=I \times F$ endowed with Lorentzian metric $\bar{g}=-\beta(t) d t^{2}+f(t) g_{F}$, where $g_{F}$ is a Riemannian metric on $F$. We can rewrite this metric as $\bar{g}=-d \tau^{2}+f(t(\tau)) g_{F}$. If we assume $f^{\prime}(t) \geq 0, f^{\prime \prime}(t) \geq 0$ and $\beta^{\prime}(t) \leq 0$, then the spacetime $M$ favors to model an accelerated expanding universe.

Our first uniqueness result is enunciated as follow (see Section 3).

Let $\left(M^{n}, \bar{g}\right), n \geq 3$, be an orthogonal-splitted spacetime. Suppose that $(M, \bar{g})$ is non-contracting (or non-expanding) in all directions. Then each compact maximal hypersurface in $M$ must be a leaf of the foliation orthogonal to the vector field $\partial_{t}$, that is, a level spacelike hypersurface of the temporal function $t$.

Some nice consequences are analized, and several applications to relevant class of spacetimes are provided.

In Section 4, we consider a change on the expansive (or contractive) behavior of the spacetime. In particular, we assume that there exists an instant $t_{0}$ of the temporal function such that the region of the spacetime $t<t_{0}$ is non-contracting in all directions, while the region $t>t_{0}$ is non-expanding in all directions. The level hypersurface $t=t_{0}$ will be called a level transition spacelike hypersurface. Then, we prove the following:

Let $\left(M^{n}, \bar{g}\right), n \geq 3$, be an orthogonal-splitted spacetime which has a level spacelike transition hypersurface. Then every compact maximal hypersurface in $M$ must be a level spacelike hipersurface.

Finally, Section 5 is devoted to present some applications to Geometric Analysis.

\section{Preliminaries}

Let $(M, \bar{g})$ be an $n+1$-dimensional orthogonal-splitted spacetime $(n \geq 2)$. An immersion $x: S \rightarrow M$ of an $n$-dimensional connected manifold $S$ into $M$ is said to be spacelike if the induced metric $g$ on $S$ is Riemannian. In this case, we will refer to $S$ as a spacelike hypersurface. For each spacelike hypersurface $S$ in $M$ we can take $N \in \mathfrak{X}^{\perp}(S)$ as the only globally unitary timelike vector field normal to $S$ in the same time-orientation of the vector field $-\partial_{t}$ (i.e., such that $\bar{g}\left(N,-\partial_{t}\right)<0$ ). From the Lorentzian metric (1), it follows that $\bar{g}\left(N, \partial_{t}\right)=\sqrt{\beta} \cosh \theta$, where $\theta$ is the hyperbolic angle between $S$ and $-\partial_{t}$ at any point of $S$ (see, for instance, [23, Prop. 5.30]). We will denote by $\tilde{t}$ the function $\pi_{I} \circ x$ on $S$, where $\pi_{I}$ is the projection of $M$ onto the time-coordinate factor.

Along this paper, the leaves of the foliation orthogonal to the vector field $\partial_{t}$ are also called level spacelike hypersurfaces. Note that a spacelike hypersurface $S$ is contained in a level spacelike hypersurface if and only if the function time $t$ is constant on $S$.

Let $\partial_{t}^{\top}:=\partial_{t}+\bar{g}\left(N, \partial_{t}\right) N$ be the tangential component of $\partial_{t}$ along $S$. It is easy to see that

$$
\nabla \tilde{t}=-\frac{1}{\beta} \partial_{t}^{\top},
$$

where $\nabla$ denotes the gradient on $S$. 
From the Gauss and Weingarten formulas associated with the immersion $x$, we have

$$
\bar{\nabla}_{X} Y=\nabla_{X} Y-g(A X, Y) N,
$$

for all $X, Y \in \mathfrak{X}(S)$, where $\nabla$ and $\bar{\nabla}$ are the Levi-Civita connections of $S$ and $M$, respectively, and $A$ is the shape operator associated to $N$,

$$
A X:=-\bar{\nabla}_{X} N .
$$

Recall that the mean curvature function relative to $N$ is given by $H:=-(1 / n) \operatorname{tr}(A)$. A spacelike hypersurface is called maximal provided that $H=0$. It is easy to check that a level spacelike hypersurface has mean curvature

$$
n H=\frac{1}{\sqrt{\beta}} \overline{\operatorname{div}}\left(\partial_{t}\right)-\frac{1}{2 \beta^{3 / 2}} \partial_{t} \beta .
$$

Thus, a a level spacelike hypersurface $\left\{t=t_{0}\right\}$, will be maximal if it satisfies the equation

$$
\frac{1}{\sqrt{\beta}} \overline{\operatorname{div}}\left(\partial_{t}\right)-\frac{1}{2 \beta^{3 / 2}} \partial_{t} \beta=0,
$$

at $t_{0}$.

On the other hand, a maximal hypersurface is a critical point of the volume variation of a certain family of hypersurfaces (see [20]). Indeed, let $x: S \longrightarrow M$ be an isometric compact spacelike hypersurface immersed into the spacetime $M$. We shall indistinctly speak of $S$ and its image if no confusion arises. Let $\omega_{S}$ be the canonical volume element of $S$ with respect to the induced metric $g$. The $n$-volume of $S$ is given by

$$
V_{S}=\int_{S} \omega_{S} .
$$

Next, let us calculate the variation of $V_{S}$ and $\omega_{S}$ when we perform a deformation of the hypersurface $S$. Let $\xi$ be a differentiable vector field defined on a neighbourhood of $x(S)$. Then, $\xi$ generates a one-parameter group $\left\{\varphi_{s}\right\}_{s \in J}$ of local transformations, where $s$ is the canonical parameter and $J \subset \mathbb{R}$ an open interval containing $s=0$. We can define a oneparameter family of isometric immersions of hypersurfaces $x_{s}: S \longrightarrow M$ given by $x_{s}=\varphi_{s} \circ x$. Observe that, in particular, $x_{0}=x$. Let us also denote by $\omega_{s}$ the corresponding canonical $n$-volume element for each $x_{s}$, being $\omega_{0} \equiv \omega_{S}$. Then, a standard calculation leads to

$$
\frac{d V_{x_{s}}}{d s}(0)=\int_{S}(\overline{\operatorname{div}} \xi+\bar{g}(\xi, \vec{H})) \omega_{0},
$$

where $\overline{\operatorname{div}}$ stands for the divergence operator of $(M, \bar{g})$. In particular, if $S$ is maximal then from the Gauss theorem it follows that $\frac{d V_{x_{s}}}{d s}(0)=0$.

\section{Spacetimes with homogeneity in its expanding-contracting behavior}

First of all, we study conditions under which an orthogonal-splitted spacetime $(M=I \times F, \bar{g})$ admits a compact spacelike hypersurface. Observe that if $F$ is compact, then the spacetime 
is spatially closed. Moreover, the converse also holds, as the following reasoning shows. Let $S$ be a (connected) compact hypersurface in the spacetime $M$ and $\left[t_{0}, t^{0}\right] \subset I$ a closed interval such that $\pi_{I}(S)=\left[t_{0}, t^{0}\right]$. Consider the canonical projection $\pi_{F}: S \longrightarrow F$.

Let $T U$ be the fibre bundle of unitary tangent vectors on $S$. Then, for all $u \in T U$ we have

$$
g(u, u) \leq g_{\pi_{I}(u)}\left(d \pi_{F}(u), d \pi_{F}(u)\right)=B(t, u) g_{t_{0}}\left(d \pi_{F}(u), d \pi_{F}(u)\right),
$$

where $B(t, u)$ is a smooth function defined on the compact interval $\left[t_{0}, t^{0}\right] \times T U$. If we denote by $\bar{B}$ the maximum of $B(t, u)$, we have

$$
g(v, v) \leq \bar{B} g_{t_{0}}\left(d \pi_{F}(v), d \pi_{F}(v)\right) .
$$

As a direct consequence the map $d \pi_{F}$ is a local dipheomorfism and, making use of [16, Lemma 3.3, Chap. 7], we conclude that $\pi_{F}$ is a covering map.

Proposition 1 i) If an orthogonal-splitted spacetime $(I \times F, \bar{g})$ admits a compact spacelike hypersurface, then $F$ is compact.

ii) Moreover, if the universal Riemannian covering of $F$ is compact and $S$ is a complete spacelike hypersurface such that the function $B(t, u)$, with $t \in \pi_{I}(S)$ and $u \in T U$, is bounded, then $S$ is compact.

This result properly extends [5, Prop. 3.2] to a wider family of spacetimes. Obviously, an orthogonal-splitted spacetime admitting a compact spacelike hypersurface is globally hyperbolic.

In order to obtain our results, next we develop a formula based on the computation of the Laplacian of the function $\tilde{t}$ on a maximal hypersurface $S$ immersed in a orthogonal-splitted spacetime $(M, \bar{g})$. From (2), a straightforward computation leads to

$$
\Delta \tilde{t}=\frac{1}{\beta^{2}} \partial_{t}^{\top}(\beta)-\frac{1}{\beta} \operatorname{div}\left(\partial_{t}^{\top}\right),
$$

where $\Delta$ and div denote the Laplacian and divergence operators of the $(S, g)$. Now, we can write

$$
\operatorname{div}\left(\partial_{t}^{\top}\right)=\overline{\operatorname{div}}\left(\partial_{t}\right)+\overline{\operatorname{div}}\left(\bar{g}\left(N, \partial_{t}\right) N\right)+\bar{g}\left(\bar{\nabla}_{N} \partial_{t}, N\right),
$$

where $\overline{\text { div }}$ denote the divergence operator of the spacetime. Since the hypersurface is maximal, we obtain

$$
\operatorname{div}\left(\partial_{t}^{\top}\right)=\overline{\operatorname{div}}\left(\partial_{t}\right)+\bar{g}\left(\bar{\nabla}_{N} \partial_{t}, N\right)
$$

The term $\overline{\operatorname{div}}\left(\partial_{t}\right)$ has a geometrical interpretation and, in fact, it can be written in terms of a certain volume function. To see that, let us take $\Omega$ the canonical Lorentzian volume element of the spacetime ([23], [3]). Then, making use of the Cartan's theorem it follows

$$
d \circ i_{\partial_{t}} \Omega=\overline{\operatorname{div}}\left(\partial_{t}\right) \Omega .
$$

Consider the level spacelike hypersurface $\left\{t=t_{0}\right\}, t_{0} \in I$, and let $\omega_{0}$ be the canonical Riemannian volume element on $\left(F, g_{t_{0}}\right)$. Since the spacetime $M$ is orthogonal-splitted, then $\Omega=\sqrt{\beta} d t \wedge \operatorname{Vol}_{\text {slice }}(t) \omega_{0}$, where the function $\operatorname{Vol}_{\text {slice }}(t)$ satisfies that $\operatorname{Vol}_{\text {slice }}(t) \omega_{0}=\omega_{t}, \omega_{t}$ 
being the canonical Riemannian volume element of the (Riemannian) manifold $\left(F, g_{t}\right)$. Hence, we have

$$
\overline{\operatorname{div}}\left(\partial_{t}\right)=\partial_{t} \log \operatorname{Vol}_{\text {slice }}+\frac{1}{2} \frac{\partial_{t} \beta}{\beta} .
$$

On the other hand, if we write the normal vector $N$ as

$$
N=-\frac{\cosh \theta}{\sqrt{\beta}} \partial_{t}+N^{F},
$$

where $\bar{g}\left(N^{F}, \partial_{t}\right)=0$, then

$$
\bar{g}\left(\bar{\nabla}_{N} \partial_{t}, \partial_{t}\right)=\frac{\cosh ^{2} \theta}{\beta} \bar{g}\left(\bar{\nabla}_{\partial_{t}} \partial_{t}, \partial_{t}\right)+\frac{\cosh \theta}{\beta}\left\{\bar{g}\left(\bar{\nabla}_{\partial_{t}} \partial_{t}, N^{F}\right)+\bar{g}\left(\bar{\nabla}_{N^{F}} \partial_{t}, \partial_{t}\right)\right\}+\bar{g}\left(\bar{\nabla}_{N^{F}} \partial_{t}, N^{F}\right)
$$

Let $p \in S$ be such that $N^{F}(p) \neq 0$ and take coordinates $\left(\mathcal{U},\left(t \equiv x_{0}, x_{1}, \ldots, x_{n}\right)\right)$ around $p$ in the product manifold $I \times F$, such that $N^{F}=\partial_{x_{1}}$ on $S \cap \mathcal{U}$. Then, from the equality which determines the Christoffel symbols for a coordinate system, we have

$$
\bar{g}\left(\bar{\nabla}_{\partial_{t}} \partial_{t}, N^{F}\right)=-\frac{1}{2} \partial_{x_{1}} \bar{g}\left(\partial_{t}, \partial_{t}\right)
$$

and

$$
\bar{g}\left(\bar{\nabla}_{N^{F}} \partial_{t}, \partial_{t}\right)=\frac{1}{2} \partial_{x_{1}} \bar{g}\left(\partial_{t}, \partial_{t}\right)
$$

Furthermore,

$$
\bar{g}\left(\bar{\nabla}_{N^{F}} \partial_{t}, N^{F}\right)=\frac{1}{2} \partial_{t} \bar{g}\left(\partial_{x_{1}}, \partial_{x_{1}}\right)
$$

Now, using (6) and (7) we get

$$
\bar{g}\left(\bar{\nabla}_{N} \partial_{t}, N\right)=-\frac{\cosh ^{2} \theta}{2 \beta} \partial_{t} \beta+\frac{1}{2} \partial_{t} \bar{g}\left(\partial_{x_{1}}, \partial_{x_{1}}\right) .
$$

Finally, from (5) we arrive to

$$
\Delta \tilde{t}=\frac{1}{\beta^{2}} \partial_{t}^{\top} \beta-\frac{1}{\beta}\left[\partial_{t} \log \operatorname{vol}_{\text {slice }}-\frac{1}{2} \frac{\sinh ^{2} \theta}{\beta} \partial_{t} \beta+\frac{1}{2} \partial_{t} \bar{g}\left(\partial_{x_{1}}, \partial_{x_{1}}\right)\right] .
$$

Now, assuming that the spacetime has dimension greater or equal than 4 , we can consider, on $S$, the metric $\tilde{g}=\beta^{2 /(n-2)} g$ pointwise conformal to the induced one $g$. In terms of this new metric, the equation (8) changes to

$$
\tilde{\Delta} \tilde{t}=-\beta^{-n /(n-2)}\left[\partial_{t} \log \operatorname{vol}_{\text {slice }}-\frac{1}{2} \frac{\sinh ^{2} \theta}{\beta} \partial_{t} \beta+\frac{1}{2} \partial_{t} \bar{g}\left(\partial_{x_{1}}, \partial_{x_{1}}\right)\right] .
$$

Therefore, if the spacetime is non-contracting or non-expanding in all directions, then for each point $q \in S$ such that $N^{F} \neq 0$ there exists an open neighborhood where $\tilde{\Delta} \tilde{t}$ have the same sign. Observe that the term $\partial_{t} \log \operatorname{vol}_{\text {slice }}$ can be physically interpreted as the increase or decrease of the local volume of the physical space for an observer in $\mathcal{U}$.

On the other hand, let $q^{\prime} \in S$ be a point where $N^{F}\left(q^{\prime}\right)=0$ and assume that there is no an open set $\mathcal{V} \in S$, with $q^{\prime} \in \mathcal{V}$, such that $N^{F}(p)=0$ for all $p \in \mathcal{V}$. As a consequence, we can take a sequence $\left\{q_{n}\right\}_{n=1}^{\infty}$ in $\mathcal{V}$ such that $\lim _{n \rightarrow \infty} q_{n}=q^{\prime}, N^{F}\left(q_{n}\right) \neq 0$ for all $n$, and $\tilde{\Delta} \tilde{t}\left(q_{n}\right)$ has equal sign for all $n$. Thus, by continuity, $\tilde{\Delta} \tilde{t}\left(q^{\prime}\right)$ has the same sign. Finally, if there exists an open set $\mathcal{V} \subset S$ such that $N^{F}(p)=0$ for all $p \in \mathcal{V}$, then $\tilde{\Delta} \tilde{t}=0$ on $\mathcal{V}$. 
Remark 2 Although this conformal change does not apply when the manifold $F$ is 2 dimensional, we can build a 1-dimensional extension in order to increase the dimension of the Riemannian factor and the spacetime. Indeed, suppose that $\operatorname{dim}(F)=2$ and let $S$ be a maximal surface in the spacetime $(I \times F, \bar{g})$. We consider the Lorentzian manifold manifold $\left((I \times F) \times \mathbb{S}^{1}, \bar{g}+d s^{2}\right)$, being $d s^{2}$ the standard metric of $\mathbb{S}^{1}$ and the following natural 3 -dimensional isometric immersion, $\hat{x}: S \times \mathbb{S}^{1} \rightarrow(I \times F) \times \mathbb{S}^{1}$, with $\hat{x}(p, s)=(x(p), s)$, for all $p \in S$ and $s \in \mathbb{S}^{1}$.

Let us consider the natural identifications

$$
T_{(p, s)}\left(S \times \mathbb{S}^{1}\right) \equiv T_{p} S \oplus T_{s} \mathbb{S}^{1}, \quad p \in S, s \in \mathbb{S}^{1}
$$

and

$$
T_{(q, s)}\left(\bar{M} \times \mathbb{S}^{1}\right) \equiv T_{q} \bar{M} \oplus T_{s} \mathbb{S}^{1}, \quad q \in \bar{M}, s \in \mathbb{S}^{1} .
$$

Then, for each tangent vector $v \in T_{p} S$ (resp. normal vector $w \in T_{p} S^{\perp}$ ) there is a canonical tangent vector $\hat{v}=(v, 0) \in T_{(p, s)}\left(S \times \mathbb{S}^{1}\right)\left(\right.$ resp. $\left.\hat{w}=(w, 0) \in T_{(p, s)}\left(S \times \mathbb{S}^{1}\right)^{\perp}\right)$. Moreover, it is clear that if the surface $S$ is maximal in $\bar{M}$, then the hypersurface $S \times \mathbb{S}^{1}$ is maximal in $\bar{M} \times \mathbb{S}^{1}$.

We are now in position to state the following result,

Theorem 3 Let $\left(M^{n}, \bar{g}\right), n \geq 3$, be an orthogonal-splitted spacetime. Assume that $(M, \bar{g})$ is non-contracting or non-expanding in all directions. Then every compact maximal hypersurface in $M$ must be a level spacelike hypersurface satisfying equation (4).

Proof. Under the assumptions in the statement above, we get from equation (9) that $\tilde{t}$ is a superharmonic or subharmonic function on a compact Riemannian manifold. Then, $\tilde{t}$ must be constant.

\section{Remark 4}

a) As equation (9) shows, if such a hypersurface $\left\{t=t_{0}\right\}$ exists, then $\partial_{t} \log \operatorname{vol}_{\text {slice }}\left(t_{0}\right)=0$.

b) A simply calculation shows that

$$
\frac{d \omega_{s}}{d s}(0)=\frac{1}{2} \operatorname{trace}_{S}\left[x^{*}\left(\mathcal{L}_{\xi} \bar{g}\right)\right] \omega_{0},
$$

where $\mathcal{L}$ stands for the Lie derivative. Assuming that the level hypersurface $\left\{t=t_{0}\right\}$ is maximal and taking $\xi=\partial_{t}$, we obtain that

$$
\frac{1}{2} \operatorname{trace}_{S}\left[x^{*}\left(\mathcal{L}_{\partial_{t}} \bar{g}\right)\right]=0 .
$$

Moreover, if $\partial_{t}$ is a conformal vector field, i.e., $\mathcal{L}_{\partial_{t}} \bar{g}=2 \rho \bar{g}$, then $\rho_{\left.\right|_{t=t_{0}}}=0$, where $\rho$ denotes the conformal factor.

Corollary 5 Let $\left(I \times F,-d t^{2}+g_{t}\right)$ be an orthogonal-splitted spacetime, with $\beta \equiv 1$, such that the physical space of the observer in $\partial_{t}$ does not expand (resp. contract) in all directions. Then every compact maximal hypersurface must be a level spacelike hypersurface. 
Remark 6 Note that, when $\beta \equiv 1$, the vector field $\partial_{t}$ is an observer field with proper time $t$, and such that the observers in $\partial_{t}$ are proper time synchronizables.

Moreover, each level spacelike hypersuperface $F(t):=\{t\} \times F$ at $t$ constant is a restspace of $\partial_{t}$, being each event in $F(t)$ simultaneous for all the observers in $\partial_{t}$.

Given $p, q \in F$, the distance between $p_{t}:=(t, p)$ and $q_{t}:=(t, q)$ measured by an observer at the instant $t$ is given by $d_{t}\left(p_{t}, q_{t}\right)$, where $d_{t}$ is the Riemannian distance in $F(t)$. Therefore, the spacetime is non-contracting (resp. non-expanding) in all directions if and only if $d_{t}\left(p_{t}, q_{t}\right)$ is non-decreasing (resp. non-increasing) for all $p, q \in F$.

We can derive some consequences from the previous results for certain relevant spacetimes. The first application is relative to multiply warped product spacetimes. Consider $I$ an interval of the real line, $m(m \geq 2)$ Riemannian manifolds $\left(F_{i}, g_{i}\right)$ and $m$ positive functions $f_{i} \in$ $C^{\infty}(I)$, and let us endow the product manifold $I \times F_{1} \times \cdots \times F_{m}$ with the Lorentzian metric $-d t^{2}+f_{1}^{2} g_{1}+\cdots+f_{m}^{2} g_{m}$.

Multiply warped products are interesting in Cosmology. For instance, it is well-known that the Kasner spacetime can be regarded as a multiply warped product [17]. Also, in the study of the $(2+1)$ BTZ Black Hole, the multiply warped products are considered [19].

Observe that, if $f_{i}=f$ for all $i$, then the spacetime is a generalized Robertson-Walker spacetime.

Corollary 7 Let $\left(I \times F_{1} \times \cdots \times F_{m},-d t^{2}+\sum_{i=1}^{m} f_{i}^{2}(t) g_{F_{i}}\right)$ be a multiply warped product spacetime, such that the functions $f_{i}^{\prime}(t)$ have a common sign for all $i=1, \ldots, n$. Then, every compact maximal hypersurface in $M$ must be a level spacelike hypersurface.

In particular, in every Lorentzian product $\left(\mathbb{R} \times F,-d t^{2}+g_{F}\right)$, the only compact maximal hypersurfaces are the spacelike level hypersurfaces.

It can also be treated the case of standard static spacetimes (compare with [30, Prop. 2]),

Corollary 8 In an $n$-dimensional $(n \geq 3)$ standard static spacetime $\left(I \times F,-h^{2} d t^{2}+g_{F}\right)$, the only compact maximal hypersurfaces are the level spacelike hypersurfaces.

Observe that, in this case, every level spacelike hypersurface $\left\{t=t_{0}\right\}$ is totally geodesic.

Finally, we can also consider the case of certain Lorentzian twisted products. Recall that a Lorentzian twisted product is a product manifold of an open interval $I$ of the real line and a Riemannian manifold $\left(F, g_{F}\right)$, endowed with the Lorentzian metric $\bar{g}=-d t^{2}+\lambda g_{F}$, where $\lambda \in C^{\infty}(I \times F)$ is a positive function. In this case, it is well-known that the level hypersurfaces at $t$ constant are totally umbilical (see [25]).

Corollary 9 In a Lorentzian twisted product spacetime $\left(I \times F,-d t^{2}+\lambda g_{F}\right)$ such that $\partial_{t} \lambda \geq$ 0 (resp. $\leq 0)$, every compact maximal hypersurface is a level spacelike hypersurface at $t$ constant.

\section{Behavior of the volume function}

In this section, we consider spacetimes whose expanding or contracting behaviour changes its monotonicity. With this aim, we introduce the following notion. We say that in an orthogonal-splitted spacetime, $\left(I \times F,-\beta d t^{2}+g_{t}\right)$, a level spacelike hypersurface $t=t_{0}$ is a 
level transition hypersurface, if the spacetime is non-contracting in the region of spacetime with $t \leq t_{0}$ and non-expanding in the region of spacetime with $t \geq t_{0}$.

Note that if there exist two different spacelike level transition hypersurfaces, then the portion of the spacetime contained between them has a geometrical and physical meaning. In geometrical terms, in this region the -metric of the- spacetime has no dependance with the $t$-coordinate; in physical terms, the spacetime does not evolve here (it is standard static in this region).

Observe that this change of behavior is similar to the one of the spatially closed Friedmann model present (see, for instance, [23, Ch. 12]).

Taking into account the formula (3), it is clear that any level transition hypersurface must be maximal.

Next, we present our second main result:

Theorem 10 Let $\left(M^{n}, \bar{g}\right), n \geq 3$, be an orthogonal-splitted spacetime which has a level spacelike transition hypersurface. Then every compact maximal hypersurface in $M$ must be also a level spacelike hypersurface.

Proof. Let $\left\{t=t_{0}\right\}$ be the level spacelike transition hypersurface. Consider the function $\tilde{t}-t_{0}$ on a compact maximal hypersurface. From (9), it follows that

$$
\left(\tilde{t}-t_{0}\right) \tilde{\Delta}\left(\tilde{t}-t_{0}\right) \geq 0 .
$$

This fact implies that $\tilde{t}-t_{0}$ is constant, and so the hypersurface must be a level spacelike hypersurface.

To clarify the last reasoning, consider any function $h \in C^{\infty}(P)$ on a compact Riemannian manifold $P$, such that $h \Delta h \geq 0$. Then, by considering $\Delta h^{2}$ and the divergence theorem, it is no difficult to see that $h$ must be constant. This, applied to the function $\tilde{t}-t_{0}$, ends the proof.

Example 11 The previous theorem can be applied to a wide family of spacetimes. For instance, let $\left(F_{i}, g_{i}\right), 1 \leq i \leq m$, be a finite set of compact Riemannian manifolds. Consider the Lorentzian multiply warped product spacetime $\left(\mathbb{R} \times F_{1} \times \cdots \times F_{m},-d t^{2}+\sum_{i} a_{i}^{2} \exp \left(-b_{i}^{2} t^{2}\right) g_{i}\right)$, where $a_{i}, b_{i}$, are non-zero constants. As a consequence of Theorem 10, in this spacetime the only compact maximal hypersurface is given by the spacelike slice $\{t=0\}$.

Remark 12 The concept of transition hypersurface can be given, in a similar way, for a spacelike hypersurface (non-necessarily for a spacelike level hypersurface). Nevertheless, such a transition hypersurface may not be maximal, as the following example shows. Consider the spacetime $\left(I \times \mathbb{S}^{1},-d t^{2}+\left(-t^{2}+2 t \sin s+3\right) d s^{2}\right)$, where $I \subset \mathbb{R}$ is the maximal domain of the $t$ coordinate where the spacetime can be defined. Here, the transition hypersurface is given by $t=\sin s$ and it is not maximal.

Remark 13 If the inequalities are reversed in the definition of transition hypersurface, counterexamples to uniqueness results can be provided. For example, consider the de Sitter spacetime, $\left(\mathbb{R} \times \mathbb{S}^{n},-d t^{2}+\cosh ^{2}(t) g_{\mathbb{S}^{n}}\right)$. Every rigid motion of the level spacelike hypersurface $\{t=0\}$ is a maximal hypersurface. Observe that, in this case, $\{t=0\}$ constitutes the level spacelike transition hypersurface with reversed behavior. 


\section{Applications to Geometric Analysis}

We will begin this section showing that every compact spacelike hypersurface in an orthogonalsplitted spacetime is a maximal hypersurface in a suitable pointwise conformal spacetime. Later, our previous uniqueness parametric results will be applied to study several Geometric Analysis problems, which arise naturally from Lorentzian Geometry.

Let $S$ be a spacelike hypersurface isometrically immersed in an orthogonal-splitted spacetime $(M, \bar{g})$. It can be considered the hypersurface $S$ isometrically immersed in the pointwise conformal spacetime $\left(M, \tilde{g}=e^{2 \alpha} \bar{g}\right)$, where $\alpha \in C^{\infty}(I \times F)$. The unitary normal vector field $N$ of $S$ in $(M, \bar{g})$, is related with the unitary normal vector field $\tilde{N}$ of $S$ in $\left(M, \tilde{g}=e^{2 \alpha} \bar{g}\right)$ by $\tilde{N}=e^{-\alpha} N$. Observe that since $S$ is spacelike in $(M, \bar{g})$, then $S$ is also spacelike in $(M, \tilde{g})$. If we take $\left\{E_{i}\right\}_{i=1}^{n}$ a local frame field on $S$ in $(M, \bar{g})$, then $\left\{e^{-\alpha} E_{i}\right\}_{i=1}^{n}$, denoted by $\left\{\tilde{E}_{i}\right\}_{i=1}^{n}$, is a local frame field on $S$ in $(M, \tilde{g})$. Denoting by $H$ and $\tilde{H}$ the mean curvatures of $S$ in $(M, \bar{g})$ and $(M, \tilde{g})$, respectively, we have

$$
n \tilde{H}=\sum_{i=1}^{n} \tilde{g}\left(\tilde{\nabla}_{\tilde{E}_{i}} \tilde{N}, \tilde{E}_{i}\right)=\sum_{i=1}^{n} g\left(\tilde{\nabla}_{E_{i}} e^{-\alpha} N, E_{i}\right)=\sum_{i=1}^{n} e^{-\alpha} g\left(\tilde{\nabla}_{E_{i}} N, E_{i}\right),
$$

where $\tilde{\nabla}$ is the Levi-Civita connection of $\tilde{g}$. Then, from the previous equation it follows

$$
e^{\alpha} \tilde{H}=H+\bar{g}(\bar{\nabla} \alpha, N) .
$$

We need the following technical result:

Lemma 14 Let $S$ be a compact spacelike hypersurface immersed in an orthogonal-splitted spacetime $(M, \bar{g})$ and $N$ its unitary normal vector field. Then, for every function $h \in C^{\infty}(S)$, there exists a function $\bar{\alpha} \in C^{\infty}(M)$ such that

$$
(\bar{\nabla} \bar{\alpha})_{\left.\right|_{S}}^{\perp}=-h N
$$

Proof. The compactness of $S$ allows to take an open interval $J$ such that the geodesics $\gamma_{p}(s)$ starting at $p \in S$ satisfying $\gamma_{p}^{\prime}(s)=N(p)$ are defined for all $p \in S$ and for all $s \in J$. On the other hand, the function $h$ can be extended to a tubular neighborhood $\mathcal{U}=\left\{\gamma_{p}(s)\right.$ : $s \in J, p \in S\}$ of $S$, being $\bar{h}$ constant along each geodesic $\gamma_{p}(s)$, with value $h(p)$. Moreover, the vector field defined in each point of $\mathcal{U}$ by $\gamma_{p}^{\prime}(s)$, also extends to the normal vector field $N$. Obviously, its flow on $I \times S$ is given by $\varphi(s, p)=\gamma_{p}(s)$, being $\varphi$ bijective. Consider the smooth function $\pi_{J} \circ \varphi^{-1}$. Then, the normal component of the gradient $\bar{\nabla}\left(\bar{h} \cdot\left(\pi_{J} \circ \varphi^{-1}\right)\right)_{\left.\right|_{S}}$ is equal to $h N$, since $\bar{g}(N, \bar{\nabla} h)=0$.

Now, let us take $\phi$ a function on $M$ such that $0 \leq \phi(p) \leq 1$, for all $p \in M$, satisfying (see Corollary in Section 1.11 of [32]) that

i) $\phi(p)=1$ if $p \in\left\{\gamma_{p}(s): s \in J^{\prime}, p \in S\right\}$, being $J^{\prime} \subset J$ a closed interval with $0 \in J^{\prime}$.

ii) $\operatorname{supp} \phi \subset \mathcal{U}$.

Therefore, the function $\phi$ allows to extend the function $\pi_{J} \circ \varphi^{-1}$ to all $M$ and obtain $\bar{\alpha}$.

Lemma 15 In an orthogonal-splitted spacetime, every compact spacelike hypersurface is a maximal hypersurface in a suitable pointwise conformal spacetime. 
Proof. It follows from equation (10) and the previous lemma.

Let $(M, \bar{g})$ be an orthogonal-splitted spacetime and $\alpha \in C^{\infty}(F)$ a smooth function. Consider the natural extension $\bar{\alpha}=\alpha \circ \pi_{F}$ on $M$. Let $S$ be a compact maximal hypersurface in $(M, \bar{g})$. Then, from (10), the mean curvature function of $S$ isometrically immersed in the conformal spacetime $\left(M, e^{2 \bar{\alpha}} \bar{g}\right)$ is given by

$$
\tilde{H}=e^{-\bar{\alpha}} \bar{g}(\bar{\nabla} \bar{\alpha}, N) .
$$

Note that the uniqueness of $S$ as maximal hypersurface in $(M, \bar{g})$, is equivalent to the uniqueness of $S$ as compact spacelike hypersurface in $\left(M, e^{2 \bar{\alpha}} \bar{g}\right)$, whose mean curvature is $\tilde{H}=e^{-\alpha} \bar{g}(\bar{\nabla} \bar{\alpha}, N)$. Making use of this fact, we can obtain some Geometric Analysis results.

Let $F$ be a compact differentiable manifold and $(I \times F, \bar{g})$ an $n$-dimensional $((\geq 4))$ orthogonal-splitted spacetime. Let $u: F \longrightarrow I$ be a smooth function, such that the entire graph $\Sigma_{u}=\{(u(p), p): p \in F\}$ is spacelike. Consider $\alpha \in C^{\infty}(F)$ and denote by $N$ the unitary normal vector field on $\Sigma_{u}$. If $\tilde{H}(u)$ stands for the mean curvature function on the graph $\Sigma_{u}$ isometrically immersed in the conformal spacetime $\left(I \times F, e^{2 \bar{\alpha}} \bar{g}\right)$, with $\bar{\alpha}=\alpha \circ \pi_{F}$, and we assume that either the spacetime $(I \times F, \bar{g})$ is non-contracting or non-expanding in all directions, or it has a level spacelike transition hypersurface, then every solution to the equation

$$
\tilde{H}(u)=e^{-\bar{\alpha}} g_{u}\left(d \pi_{F}(N), D_{u} \alpha\right),
$$

being $D_{u}$ the gradient operator in $\left(F, g_{u}\right)$, must be constant.

This last fact can be particularized to the case in which the Lorentzian ambient manifold is a generalized Robertson-Walker spacetime.

Let $\left(F, g_{F}\right)$ be a $n$-dimensional compact Riemannian manifold and $f: I \longrightarrow \mathbb{R}$ a positive smooth function. For each $u \in C^{\infty}(F)$ such that $u(F) \subseteq I$, we can consider its graph $\Sigma_{u}=\{(u(p), p): p \in F\}$ in the Lorentzian warped product $\left(M=I \times_{f} F, \bar{g}\right)$. The graph inherits from $M$ a metric, represented on $F$ by

$$
g_{u}=-d u^{2}+f(u)^{2} g_{F} .
$$

This metric is Riemannian (i.e. positive definite) if and only if $u$ satisfies $|D u|<f(u)$ everywhere on $F$, where $D u$ denotes the gradient of $u$ in $\left(F, g_{F}\right)$ and $|D u|^{2}=g_{F}(D u, D u)$. Note that $\tilde{t}(u(p), p)=u(p)$ for any $p \in F$, and so $\tilde{t}$ and $u$ may be naturally identified on $\Sigma_{u}$.

When $\Sigma_{u}$ is spacelike, the unitary normal vector field on $\Sigma_{u}$ satisfying $\bar{g}\left(N, \partial_{t}\right)<0$ is

$$
N=\frac{1}{f(u) \sqrt{f(u)^{2}-|D u|^{2}}}\left(f(u)^{2} \partial_{t}+D u\right) .
$$

Theorem 16 Let $\left(F, g_{F}\right)$ be a compact Riemannian manifold, and $\alpha: F \longrightarrow \mathbb{R}, f: I \longrightarrow$ $(0, \infty)$ smooth functions, where $I=(a, b)$ is an open interval, satisfying:

i) either $f^{\prime}$ is signed,

ii) or $f$ is non-decreasing in the interval $\left(a, t_{0}\right)$ and non-increasing in the interval $\left(t_{0}, b\right)$, for some $t_{0} \in(a, b)$.

Then, the only entire spacelike graphs $\Sigma_{u}=\{(u(p), p): p \in F\}$ in the spacetime $(I \times$ $F,-e^{2 \bar{\alpha}} d t^{2}+e^{2 \bar{\alpha}} g_{F}$ ) whose mean curvature $\tilde{H}$ is prescribed by 


$$
\tilde{H}(u)=\frac{e^{-\bar{\alpha}}}{f(u) \sqrt{f(u)^{2}-|D u|^{2}}} g_{F}(D \alpha, D u)
$$

are the constant graphs $u=u_{0}$ such that $f^{\prime}\left(u_{0}\right)=0$.

Note that the graph $\Sigma_{u}$ is spacelike in $\left(I \times F,-e^{2 \bar{\alpha}} d t^{2}+e^{2 \bar{\alpha}} g_{F}\right)$ if and only if so is in $\left(I \times F,-d t^{2}+f(t)^{2} g_{F}\right)$. On the other hand, if we conceive the mean curvature function of a graph $\Sigma_{u}$ as a known operator $\tilde{H}(u)$ on $C^{\infty}(F)$, Theorem 16 can be rewritten in the following equivalent form:

Theorem 17 Let $\left(F, g_{F}\right)$ be a compact Riemannian manifold, and $\alpha: F \longrightarrow \mathbb{R}, f: I \longrightarrow$ $(0, \infty)$ smooth functions, where $I=(a, b)$ is an open interval, satisfying:

i) either $f^{\prime}$ is signed,

ii) or $f$ is non-decreasing in the interval $\left(a, t_{0}\right)$ and non-increasing in the interval $\left(t_{0}, b\right)$, for some $t_{0} \in(a, b)$.

Then, the only solutions to the differential equation

$$
\tilde{H}(u)=\frac{e^{-\alpha}}{f(u) \sqrt{f(u)^{2}-|D u|^{2}}} g_{F}(D \alpha, D u),
$$

with the restriction $|D u|<f(u)$ are the constant functions $u=u_{0}$, with $f^{\prime}\left(u_{0}\right)=0$.

\section{Acknowledgments}

Juan A. Aledo was partially supported by MINECO/FEDER Grant no. MTM2016-80313P. Rafael M. Rubio is partially supported by Spanish MINECO and ERDF Project No. MTM2016-78807-C2-1-P.

\section{References}

[1] J.A. Aledo, A. Romero and R.M. Rubio, Constant mean curvature spacelike hypersurfaces in Lorentzian warped products and Calabi-Bernstein type problems, Nonlinear Anal. 106 (2014), 57-69.

[2] J.A. Aledo, R.M. Rubio and J.J. Salamanca, Complete spacelike hypersurfaces in generalized Robertson-Walker and the null convergence condition, Calabi-Bernstein problems, J.J. RACSAM 111 (2017), 115-128.

[3] J.A. Aledo, A. Romero and R.M. Rubio, Upper and lower bounds for the volume of a compact spacelike hypersurface in a generalized RobertsonWalker spacetime, International Journal of Geometric Methods in Modern Physics 11 n 01 (2014), p. 1450006.

[4] L.J. Alías and S. Montiel, Uniqueness of spacelike hypersurfaces with constant mean curvature in generalized Robertson-Walker spacetimes. Differential geometry, Valencia, 2001, 5969, World Sci. Publ., River Edge, NJ, (2002).

[5] L.J. Alías, A. Romero and M. Sánchez, Uniqueness of complete spacelike hypersurfaces of constant mean curvature in Generalized Robertson-Walker spacetimes, Gen. Relativity Gravitation 27 (1995), 71-84. 
[6] R. Bartnik, Existence of maximal surfaces in asymptotically flat spacetimes, Comm. Math. Phys. 94 (1984), 155-175.

[7] J.K. Beem, P.E. Ehrlich and K.L. Easley 1996 Global Lorentzian Geometry (Pure and Applied Mathematics vol 202) 2nd edn (New York: Dekker)

[8] A.N. Bernal and M. Sánchez, Smoothness of time functions and the metric splitting of globally hyperbolic spacetimes, Com. Math. Phys. 257 (2005), 43-50.

[9] D. Brill, Maximal Surfaces in Closed and Open Spacetimes, Proceedings on the first Marcel Grossman meeting on General Relativity (1977), 193-206.

[10] D. Brill and F. Flaherty, Isolated maximal surfaces in spacetime, Comm. Math. Phys. 50 (1984), 157-165.

[11] E. Calabi, Examples of Bernstein problems for some non-linear equations, Proc. Sympos. Pure Math. 15 (1970), 223-230.

[12] S.Y. Cheng, S.T. Yau, Maximal space-like hypersurfaces in the Lorentz-Minkowski spaces, Ann. Math. 104 (1976), 407-419.

[13] Y. Choquet-Bruhat and R. Geroch, Global Aspects of the Cauchy Problem in General Relativity, Commun. Math. Phys. 14 (1969), 329-335.

[14] Y. Choquet-Bruhat, The problem of constraints in General Relativity: solution of the Lichnerowicz equation. Differential Geometry and Relativity, Math. Phys. Appl. Math. vol. 3, D. Reidel, Dordrecht (1976), 225-235.

[15] Y. Choquet-Bruhat, General Relativiy and the Einstein Equations, Oxford Math. Monogr., Oxford Univ. Press, New York, 2009.

[16] M. Do Carmo, Riemannian Geometry, Birkäuser, Boston 1992.

[17] A. Harvey, Geodesics in Kasner universes, Phys. Rev. D 39 (1989), 673-676.

[18] S.W. Hawking, The existence of Cosmic Time Functions, Proc. Roy. Soc.. London, Series A 308 (1969), 433-435.

[19] S-T. Hong, J. Choi and Y-J. Park, $(2+1)$ BTZ Black Hole and Multiply Warped Product Spacetimes, Gen. Relativity Gravitation 35 (2003), 2105-2116.

[20] J. Josr, Riemannian Geometry and Geometric Analysis, Springer, New York 2010.

[21] J.E. Marsden and F.J. Tipler, Maximal hypersurfaces and foliations of constant mean curvature in General Relativity, Phys. Rep. 66 (1980), 109-139.

[22] H. Omori, Isometric immersions of Riemannian manifolds, J. Math. Soc. Japan 19 (1967), 205-214.

[23] B. O'Neill, Semi-Riemannnian Geometry, Academic Press, New York (1983).

[24] J.A.S. PelegrÃn, A. Romero, Rubio, Rafael M., Uniqueness of complete maximal hypersurfaces in spatially open $(n+1)$-dimensional Robertson-Walker spacetimes with flat fiber, Gen. Relativity Gravitation 48 (2016), 14 pp. 
[25] R. Ponge and H. Reckziegel, Twisted products in pseudo-Riemannian geometry, Geom. Dedicata 48 (1993), 15-25.

[26] A. Romero, R.M. Rubio and J.J. Salamanca, Uniqueness of complete maximal hypersurfaces in spatially parabolic Generalized Robertson-Walker spacetimes, Class. Quantum Grav. 30 (2013), 115007 (13pp).

[27] A. Romero, R. Rubio and J.J. Salamanca, A new approach for uniqueness of complete maximal hypersurfaces in spatially parabolic GRW spacetimes, J. Math. Anal. Appl., to appear.

[28] A. Romero, R. Rubio and J.J. Salamanca, Complete maximal hypersurfaces in certain spatially open generalized Robertson-Walker spacetimes, J.J. RACSAM, 109 (2015), 451-460.

[29] M. Sánchez, Recent progress on the notion of global hyperbolicity, Chapter in Advances in Lorentzian geometry Amer. Math. Soc. 49 (2011), 105-124.

[30] M. Sánchez and M.M. Senovilla, A note on the uniqueness of global static decompositions, Class. Quantum Grav. 24 (2007), 6121-6126.

[31] R. Schoen and S.-T. Yau, On the proof of the possitive mass conjeture in General Relativity, Comm. Math. Phys. 65 (1979), 45-76.

[32] F.W. Warner, Foundations of Differentiable Manifolds and Lie Groups, Springer 1983, New York.

[33] S.T. Yau, Harmonic functions on complete Riemannian manifolds, Comm. Pure Appl. Math. 28 (1975), 201-228. 